\title{
A formulation of a multi-wave elastodynamic infinite element
}

\author{
K. Kazakov \\ Department of Structural Mechanics, VSU “Luben Karavelov”, Sofia, \\ Bulgaria
}

\begin{abstract}
In this paper, a formulation of a multi-wave elastodynamic four and eight node infinite element is proposed. Such a kind of element is appropriate for multi-wave soil-structure interaction problems. The formulation follows the standard infinite element formulation steps which are the same as for the Finite element method after mapping the infinite element domain to a finite element domain. It is shown that if only one wave function is used (only one frequency) the proposed multi-wave elastodynamic infinite element is reduced to a singlewave elastodynamic infinite element.

The mapping and the Lagrange isoparametric shape functions for a $2 D$ axisymmetric four and eight node multi-wave elastodynamic quadrilateral infinite element are also given. The basic aspects of the continuity along the finite/infinite element (artificial boundary) line are discussed in brief. In this type of model such a line marks the boundary between the near and the far field of the model.

The formulation is appropriate for wave propagation problems only.

Keywords: wave propagation, infinite elements, finite element method, soil-structure interaction.
\end{abstract}

\section{Introduction}

This section is devoted to the review of the historical background of infinite elements from the original works to the latest contribution. Exterior domain scattering problems appear in many engineering fields such as electrodynamics, magnetics, fluid and thermal analyses and so on. Wave propagation in an elastic infinite media and scattering of waves on bodies in a fluid which extends 
infinitely are of particular interest. The difficulty in such problems when numerical methods are used arises from the unbounded domain that has to be discretized. Many suggestions and ideas for the treatment of the exterior domain have been presented and discussed in a number of research papers for the period of three decades. The exterior (infinite) domain cannot be discretized with standard finite elements. A lot of efforts have been spent in the development of new infinite element formulations and techniques, based on the changes of the polynomial shape functions with trigonometric or exponential forms.

In soil-structure interaction problems one possible approach is just to truncate the computational domain at some distance (line) away and to impose some "appropriate" boundary conditions. Such a line is called an "artificial" boundary. In this case viscous, absorbing or transmitting boundary conditions can be used. It is evident that the computational efficiency depends then on the localization of the "artificial" boundary and the type of the boundary conditions. In many cases such techniques give acceptable results. In soil-structure interaction problems that approach is known as the Substructural approach.

\section{Earlier infinite element method works}

The infinite element method was introduced about three decades ago in the original work of Bettess [5]. Then this method have been developed and refined in many works. Between them are the works of Pissanetzky on the magnetostatics and Kim on the magnetic field problems. The original Bettess formulation is similar to the finite element concept except the element domain. In this formulation the domain extends toward infinity in one direction. The corresponding shape functions are analytically integrable over the element. Such an infinite element is directly applicable to the Finite element method.

The mapped infinite elements were developed by Zienkiewicz et al. [18]. These elements are based on polynomial shape functions, attenuating in the infinity. The mapping technique assures direct integration.

A mathematically precise variational formulation of infinite elements has only recently been proposed [15].

\section{Practical classification of infinite elements}

From a practical point of view infinite elements can be classified into five classes:

- classical infinite elements,

- decay infinite elements,

- $\quad$ mapped infinite elements,

- $\quad$ elastodynamic infinite elements and

- Wave envelope infinite elements.

The origin of the idea and the development of every one of the above classes are difficult to be dated. The first class is based on the original so-called "classical" formulation of the infinite elements. In the decay, infinite element 
decay functions from different mathematical types are used. The mapped infinite elements are developed by usage of mapping techniques. These techniques map the infinite domain of the element into a finite. The obtained element is similar to the classical finite element. The latest researches of infinite elements are devoted to the development of the elastodynamic infinite elements and the wave envelope infinite elements. In some cases the last two classes can be considered as a special combination of the mapped and decay infinite elements.

\section{Multi-wave elastodynamic infinite element formulation}

The displacement field in the multi-wave elastodynamic infinite element can be described in the standard form by a finite number of shape functions based on wave propagation functions [6] as

$$
\mathbf{u}(x, z, \omega)=\sum_{i=1}^{n} \sum_{q=1}^{m} N_{i q}(x, z, \omega) \mathbf{p}_{i q}(\omega)
$$

or

$$
\mathbf{u}(x, z, \omega)=\mathbf{N}_{p}(x, z, \omega) \mathbf{p}(\omega)
$$

where $N_{i q}(x, z, \omega)$ are the standard shape displacement functions, $\mathbf{p}_{i q}(\omega)$ are the generalized coordinates associated with $N_{i q}(x, z, \omega), n$ is the number of nodes for the element and $m$ is the number of wave functions included in the formulation of the infinite element. For horizontal wave propagation the shape displacement functions can be expressed as:

$$
N_{i q}(x, z, \omega)=L(\eta) W_{q}(\xi, \omega)
$$

where $W_{q}(\xi, \omega)$ are wave functions related to a horizontal propagation (in $\xi$ direction) and $L(\eta)$ is a Lagrange polynomial. The infinite element domain is shown in fig.1. By taking into account only the real parts of the wave functions, the equations of the wave propagation can be written as

$$
\operatorname{Re} W_{q}(\xi, \omega)=\cos \left(\frac{i \omega}{c_{s}} \xi\right) e^{-\alpha \xi}
$$

or

$$
\operatorname{Re} W_{q}(\xi, \omega)=\cos \left(\frac{i \omega}{c_{p}} \xi\right) e^{-\alpha \xi}
$$

where $c_{s}$ and $c_{p}$ are the velocities of the $S$-waves and $P$-waves respectively. 


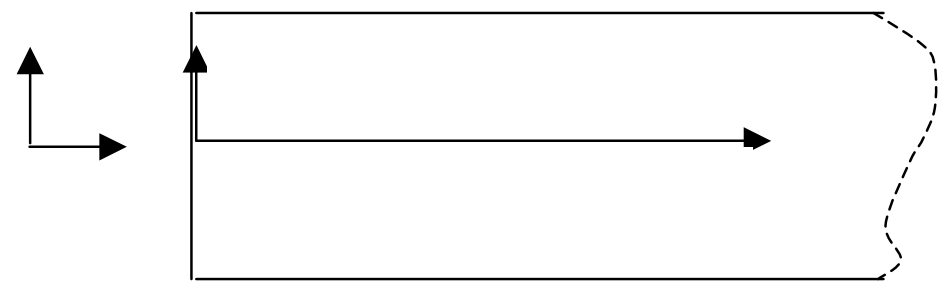

Figure 1: The infinite element domain.

If the number $m$ is known these functions can be collected preliminary as

$$
\operatorname{Re} W(\xi)=\frac{1}{m} \sum_{q=1}^{m} A_{q} \cos \left(\frac{i \varpi q}{c_{s}} \xi\right) e^{-\alpha \xi}
$$

or

$$
\operatorname{Re} W(\xi)=\frac{1}{m} \sum_{q=1}^{m} A_{q} \cos \left(\frac{i \varpi q}{c_{p}} \xi\right) e^{-\alpha \xi}
$$

where $\varpi$ is the lowest frequency and $\omega=\varpi q$. The coefficients $A_{q}$ can be written as:

$$
A_{q}=\int_{0}^{T_{q}} \operatorname{Re} W(\xi, \omega) \cos \left(\frac{i \varpi q}{c_{S}} \xi\right) d t
$$

Now so-called united shape function can be written as

$$
N_{i}(x, z)=\sum_{q=1}^{m} N_{i q}(x, z, \omega)=L(\eta) \operatorname{Re} W(\xi)
$$

Finally equation (1) can be expressed as

$$
\mathbf{u}(x, z, t)=\sum_{i=1}^{n} N_{i}(x, z, t) \mathbf{p}_{i}(t)
$$

or

$$
\mathbf{u}(x, z)=\mathbf{N}_{p}(x, z) \mathbf{p}
$$


The procedure described by the equations (4)-(6) can be treated as a superposing procedure based on a finite number of terms, where the real components of the wave functions $\operatorname{Re} W_{q}(\xi, \omega)$ are called "preliminary" shape functions. The coefficients $A_{q}$ are generalized coordinates with only one component, corresponding to the node $i$.

It is easy to be shown that in the case of only one wave function used in the computational model, only one frequency, the proposed multi-wave elastodynamic infinite element is reduced to a single-wave elastodynamic infinite element. It can be treated as a special case. Then

$$
N_{i}(x, z)=N_{i q}(x, z, \omega)=L(\eta) \operatorname{Re} W(\xi, \omega)
$$

or

$$
N_{i}(x, z)=N_{i q}(x, z)=L(\eta) \operatorname{Re} W(\xi) .
$$

\section{Two dimensional mapped infinite element}

The next step is to generate mapping to map the infinite element domain to a finite domain and vice versa. Mapping functions and the Lagrange isoparametric shape functions for a $2 D$ axisymmetric four node quadrilateral mapping infinite element and for a $2 D$ axisymmetric eight node quadrilateral mapping infinite element can be written as follows.

\section{$5.12 D$ axisymmetric four node quadrilateral mapping infinite element}

\subsubsection{Mapping functions}

$$
\begin{aligned}
& x=x_{I} \frac{(1-\eta)(-\xi)}{1-\xi}+x_{J} \frac{(1+\eta)(-\xi)}{1-\xi}+\frac{1}{2} x_{K} \frac{(1+\eta)(1+\xi)}{1-\xi}+ \\
& +\frac{1}{2} x_{L} \frac{(1-\eta)(1+\xi)}{1-\xi} \\
& y=y_{I} \frac{(1-\eta)(-\xi)}{1-\xi}+y_{J} \frac{(1+\eta)(-\xi)}{1-\xi}+\frac{1}{2} y_{K} \frac{(1+\eta)(1+\xi)}{1-\xi}+ \\
& +\frac{1}{2} y_{L} \frac{(1-\eta)(1+\xi)}{1-\xi}
\end{aligned}
$$

\subsubsection{Lagrange isoparametric shape functions (displacement field)}

$$
\begin{aligned}
& u=\frac{1}{4} u_{I}(1-\eta)\left(\xi^{2}-\xi\right)+\frac{1}{4} u_{J}(1+\eta)\left(\xi^{2}-\xi\right)+ \\
& +\frac{1}{4} u_{K}(1+\eta)\left(1-\xi^{2}\right)+\frac{1}{4} u_{L}(1-\eta)\left(1-\xi^{2}\right)
\end{aligned}
$$




\section{$5.22 D$ axisymmetric eight node quadrilateral mapping infinite element}

\subsubsection{Mapping functions}

$$
\begin{aligned}
& x=x_{I} \frac{(1-\eta)(-1-\xi-\eta)}{1-\xi}+2 x_{J} \frac{\left(1-\eta^{2}\right)}{1-\xi}+x_{K} \frac{(1+\eta)(-1-\xi+\eta)}{1-\xi}+ \\
& +\frac{1}{2} x_{L} \frac{(1+\eta)(1+\xi)}{1-\xi}+\frac{1}{2} x_{M} \frac{(1+\eta)(1+\xi)}{1-\xi} \\
& y=y_{I} \frac{(1-\eta)(-1-\xi-\eta)}{1-\xi}+2 y_{J} \frac{\left(1-\eta^{2}\right)}{1-\xi}+y_{K} \frac{(1+\eta)(-1-\xi+\eta)}{1-\xi}+ \\
& +\frac{1}{2} y_{L} \frac{(1+\eta)(1+\xi)}{1-\xi}+\frac{1}{2} y_{M} \frac{(1+\eta)(1+\xi)}{1-\xi}
\end{aligned}
$$

\subsubsection{Lagrange isoparametric shape functions (displacement field)}

$$
\begin{aligned}
& u=\frac{1}{4} u_{I}(1-\eta)(1-\xi)(-1-\eta-\xi)+\frac{1}{2} u_{J}\left(1-\eta^{2}\right)(1-\xi)+ \\
& +\frac{1}{4} u_{K}(1+\eta)(1-\xi)(-1+\eta-\xi)+\frac{1}{2} u_{L}(1+\eta)\left(1-\xi^{2}\right)+ \\
& +\frac{1}{2} u_{M}(1-\eta)\left(1-\xi^{2}\right)
\end{aligned}
$$

\subsection{Mass and stiffness matrices}

The stiffness and mass matrices can be given in a standard of the Finite element method form as

$$
\left[k_{e}\right]=\int_{\Omega_{e}}[B]^{T}[D][B] d \Omega_{e} \text { and }\left[m_{e}\right]=\left(\int_{\Omega_{e}}[N]^{T}[N] d \Omega_{e}\right) I
$$

where $[N]$ are the shape functions and the vectors $\left\{B_{i}\right\}$ in the matrix $[B]$ are written as

$$
\left\{B_{i}\right\}=\left\{\begin{array}{l}
\frac{\partial N_{i}}{\partial x} \\
\frac{\partial N_{i}}{\partial y}
\end{array}\right\} \quad \text { or } \quad\left\{\bar{B}_{i}\right\}=[J]^{1}\left\{\begin{array}{l}
\frac{\partial \bar{N}_{i}}{\partial \xi} \\
\frac{\partial \bar{N}_{i}}{\partial \eta}
\end{array}\right\}
$$


where $[J]^{1}$ is the Jacobian matrix which defines the geometrical mapping and can be written as

$$
[J]^{1}=\left[\begin{array}{ll}
\frac{\partial \xi}{\partial x} & \frac{\partial \eta}{\partial x} \\
\frac{\partial \xi}{\partial y} & \frac{\partial \eta}{\partial y}
\end{array}\right]
$$

The domain differential $d \Omega_{e}$ must also be written in terms of the local coordinates as

$$
d \Omega_{e}=d x d y=[J] d \eta d \xi
$$

Subject to the evaluation of $\left\{B_{i}\right\}$ and $d \Omega_{e}$, which involves the mapping functions, the element stiffness and mass matrices may not be computed with standard Gaussian procedure.

\section{Continuity through finite and infinite elements}

The continuity through finite and infinite elements can be enforced in exactly the same way as between two finite elements in the case they have the same degrees of freedom and the degree of approximation. A sketch of the boundary between finite and infinite elements is given in fig. 2.

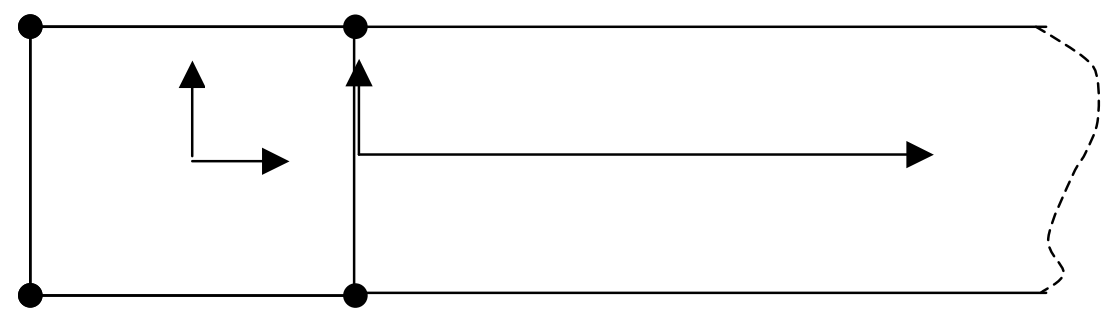

Figure 2: $\quad$ Sketch of the boundary between finite and infinite elements.

\section{Conclusion}

This paper proposes a formulation of a multi-wave elastodynamic infinite element, appropriate for multi-wave soil-structure interaction problems. In the case of only one included wave function, the proposed multi-wave elastodynamic infinite element is reduced to a single-wave elastodynamic infinite element. 
The formulation follows the standard infinite element formulation steps which are the same as in the Finite element method after the mapping the infinite domain to a finite domain of the element.

Also the mapping and the Lagrange isoparametric shape functions for a $2 D$ axisymmetric four node multi-wave elastodynamic quadrilateral infinite element and for a $2 D$ axisymmetric eight node multi-wave elastodynamic quadrilateral infinite element are given.

\section{References}

[1] Wolf J.P, Song C, Finite-element modeling of unbounded media. Willey, 1996.

[2] Bathe K.J., Finite element procedures in engineering analysis. PrenticeHill, 1982.

[3] Bancov B. P., Palova J. B., "The Finite Element Method in Structural Mechanics", UACG, 1996, (in Bulgarian).

[4] Ungless R.F., "Un infinite elements”, M.A. Sc. Dissertation, University of British Columbia, 1973.

[5] Bettess P., "Infinite elements", International Journal for Numerical Methods in Engineering, 1978; 11:54-64.

[6] Yan Ch. B., Kim D.K., Kim J.N., “Analytical frequency-dependent infinite elements for soil-structure interaction analysis in two-dimensional medium", Engineering Structures 22 (2000); 258-271.

[7] Wolf J.P., "Soil-Structure Interaction Analysis in Time Domain", Englewood Cliffs, N.J.: Prentice-Hill, 1988.

[8] Kazakov K, "A model of one-dimensional wave propagation in homogeneous media”, Journal Stroitelstvo, 6/2004, 12-14. (in Bulgarian)

[9] Kazakov K, "An adequate computational model of the infinite soil for Soil-Structure Interaction Problems", Proceedings of X Congress of applied mechanics, Bulgarian Academy of Science, Varna, Bulgaria, 2005.

[10] Kazakov K, "On the model of elastodynamic infinite element for the far field in Soil-Structure Interaction problems", Proceedings of National conference with international participation of VSU "Liuben Karavelov", Sofia, 2005, (in Bulgarian).

[11] Kazakov K, "A model if FEM type elastodynamic infinite element for Soil-Structure Interaction", Proceedings of the 4th International Conference on New trends in Static and Dynamics of structure 20-21 October 2005. Bratislava, Slovakia.

[12] Kazakov K., "Continuity between Finite and Infinite Elements, Along Artificial Boundary in Soil-Structure Interaction Problems", Proceedings of the Jubilee Conference in UACG 2007, Sofia, Bulgaria

[13] Madabhushi S. P. G., "Modeling of deformations in Dynamic SoilStructure Interaction problems", VELACS, 1996.

[14] Park K. L., Watanabe E., Utsunomiya T., "Development of 3D elastodynamic infinite elements for Soil-Structure Interaction Problems", 
International Journal of Structural Stability and Dynamics, Vol. 4, No. 3 (2004) 423-441

[15] Cecot W., Demkowicz L., Rachowicz W., "A three-dimensional infinite element for Maxwell's equations", TICAM Report 00.20

[16] Gerdes K., "A review of Infinite Element Method", Journal of Computational Acoustics

[17] Zhao Ch., Valliappan S., "A Dynamic Infinite Element for Threedimensional Infinite Domain Wave Problems", International Journal for Numerical Methods in Engineering, Vol. 36, (1993), 2567-2580

[18] Zienkievicz O. C., Bando K., Bettess P., Emson C., Chiam T. C., "Mapped Infinite Elements for Exterior Wave Problems", International Journal for Numerical Methods in Engineering, Vol. 21, (1985), 12291251 